\title{
Emergency contraception in Australia: the desired source of information versus the actual source of information
}

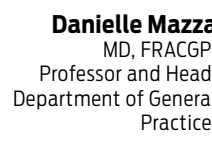

Christopher M

Harrison

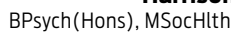
Senior Research Analyst

Angela J Taft

BA, MPH, PhD

Professor and Director ${ }^{3}$

Helena C Britt BA, PhD,

Associate Professor and

Director $^{2}$

Melissa Hobbs

RN, MPH, PhD

Manager, Population

Health

Kay Stewart

BPharm(Hons), PhD, Associate Professor, Centre

for Medicine Use and

Safety

Safeera Hussainy BPharm(Hons), PhD, GCHE, Lecturer, Centre for

Medicine Use and Safety

Bianca R Brijnath

BA(Hons), PhD, NHMRC

Early Career Fellow.

Department of General

Practice

1 Monash University, Melbourne, VIC.

2 Family Medicine Research Centre, University of Sydney, Sydney, NSW.

3 Judith Lumley Centre, La Trobe University, Melbourne, VIC

4 ACT Medicare Local, Canberra, ACT.

danielle.mazza@ monash.edu

MJA 2014; 200: 414-415 doi: 10.5694/mjal3.10983

\section{$T$}

he emergency contraceptive pill (ECP) has been available over the counter (OTC) to Australian women from pharmacies for almost a decade. Yet the rate of unplanned pregnancy remains high, ${ }^{1}$ and nearly half of all Australian women of reproductive age have experienced an unplanned pregnancy. ${ }^{1}$ Similar findings have been reported from the United Kingdom, ${ }^{2}$ the United States ${ }^{3}$ and Canada. ${ }^{4}$ This suggests that the potential for emergency contraception (EC) to reduce the rate of unwanted pregnancies is still unrealised.

Australian research shows that although women have a high general awareness of the ECP, they want more information on how to procure and use it, and they prefer to receive this knowledge from their general practitioner. ${ }^{5}$ However, with the availability of the ECP OTC from 2004 onwards, it is likely that fewer women of reproductive age would consult their GP for EC management. Therefore, the aim of this paper is to determine the extent of the change of EC management in general practice and what implications such change poses for women's sexual and reproductive health.

\section{Methods}

Data from the BEACH (Bettering the Evaluation and Care of Health) program, collected between April 2000 and March 2012, were analysed. $\mathrm{BEACH}$ is a continuous, paper-based, national study of GP activity in Australia. ${ }^{6}$ Each year, the 1000 GPs in the national, rolling random sample each provides information on 100 consecutive encounters with consenting, unidentified patients. Each year, the study runs from April to March of the following year. The University of Sydney Human Research Ethics Committee and the Australian Institute of Health and Welfare Ethics Committee have approved BEACH.

\begin{abstract}
Objective: To determine long-term trends in emergency contraception (EC) management by general practitioners in Australia.

Design, setting and participants: Data from April 2000 to March 2012 were drawn from the BEACH (Bettering the Evaluation and Care of Health) program, a continuous cross-sectional survey of GP activity. We analysed consultations involving EC management, unwanted pregnancy management and emergency contraceptive pill (ECP) prescribing per $1000 \mathrm{GP}$ encounters with women aged 14-54 years. Summary statistics were calculated with $95 \%$ confidence intervals.
\end{abstract}

Results: In 2000-2001, GPs managed EC problems at a rate of 5.50 per 1000 encounters ( $95 \% \mathrm{Cl}, 4.37-6.63)$. From 2004, after the ECP became available over the counter (OTC) in pharmacies, EC management, which includes ECP prescription, progressively declined. By 2011-2012, only 1.43 EC problems were managed per 1000 encounters $(95 \% \mathrm{Cl}, 0.84-2.02)$ and only $0.48 \mathrm{ECP}$ prescriptions were provided per 1000 encounters ( $95 \% \mathrm{Cl}, 0.14-0.82)$. Yet the management rate of unwanted pregnancy problems stayed relatively constant (rate in 2000-2001, 0.95 per 1000 encounters; 95\% Cl, 0.40-1.50; rate in 20112012, 0.88 per 1000 encounters; $95 \% \mathrm{Cl}, 0.41-1.36$ ).

Conclusion: Low rates of EC management by GPs since ECP became available OTC suggest that women may be obtaining information on EC elsewhere. Further investigation is needed to uncover the sources of this information and its acceptability and application by Australian women.

We analysed encounters with female patients of reproductive age (defined as age $14-54$ years) and examined EC management including ECP prescription, unwanted pregnancy management, procedures undertaken and referrals. Problems managed at encounters were classified according to the International classification of primary care, 2nd edition $(\text { ICPC }-2)^{7}$ and coded to more specific ICPC-2 PLUS terminology. ${ }^{8}$ EC was defined as including "postcoital contraception" (W10), "fear of pregnancy" (W02) and "unprotected sex, female" (A23011). Unwanted pregnancy was defined as "unwanted pregnancy" (W79) and "induced abortion" (W83).

The BEACH study uses a singlestage cluster design, with a cluster of encounters around each GP. In all analyses, we adjusted for this cluster using survey procedures in SAS (SAS Institute Inc). Statistical significance of differences was determined by non-overlapping $95 \%$ confidence intervals. The statistical reliability and validity of $\mathrm{BEACH}$ has been established. ${ }^{9}$

\section{Results}

BEACH data showed that in 20002001, before the introduction of the ECP OTC, GPs managed emergency contraception (EC) problems at a rate of 5.50 per 1000 encounters with women aged $14-54$ years $(95 \%$ CI, 4.37-6.63). This rate of EC management (which may or may not include the ECP prescription) was unchanged (5.38 per 1000 encounters; 95\% CI, 4.23-6.53) when ECP prescriptions were at their peak in 2003-2004 (rate of ECP prescriptions, 4.78 per 1000 encounters; 95\% CI, 3.65-5.91).

However, by 2011-2012, EC problems were managed at a rate of only 1.43 per 1000 encounters with women aged $14-54$ years $(95 \%$ CI, $0.84-2.02)$, and ECP prescriptions were provided at a rate of only 0.48 per 1000 encounters $(95 \% \mathrm{CI}, 0.14-0.82)$. The initial significant drop in EC management occurred when the ECP was made available OTC from 2004.

Since then, rates of EC management have progressively declined in general practice, while rates of unplanned pregnancy have stayed rel- 
Rate of emergency contraception management, unwanted pregnancy management and emergency contraceptive pill (ECP) prescribing* per 1000 general practitioner encounters with women aged $14-54$ years $^{\dagger \ddagger}$

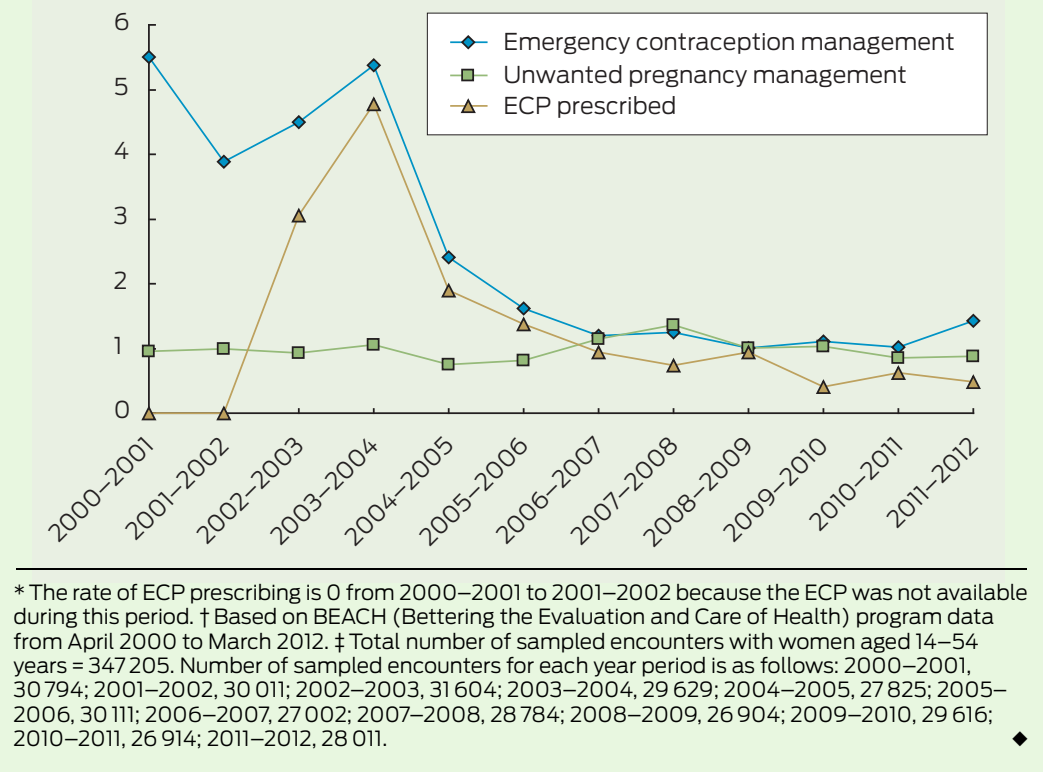

atively constant (Box). BEACH data show that the management rate of unwanted pregnancy problems per 1000 encounters with women aged 14-54 years was 0.95 (95\% CI, 0.40 1.50) in 2000-2001 and 0.88 (95\% CI, $0.41-1.36)$ in 2011-2012.

\section{Discussion}

The progressive decline of EC management in general practice since the introduction of the ECP OTC in 2004 suggests that women may be obtaining information on EC elsewhere. These other sources may include the internet or pharmacists when women purchase the ECP OTC. Yet such sources may not necessarily be the preferred source of information. Recent research shows that Australian women prefer to receive knowledge about the ECP from their GP. ${ }^{5}$

One of the reasons for this preference may be because of the environment in which such information is communicated. Private consultations may be preferred over other more "open" settings and this preference may apply to women and their health providers. Pharmacists, for example, prefer to counsel women on the ECP and other forms of contraception when confidentiality can be assured and in a separate area away from other customers. ${ }^{10}$ However, large or busy pharmacies may be less conducive environments for such health education to occur.

Other reasons for the decreasing rate of EC management in general practice could be because of better delivery and uptake of other forms of contraception. However, our recent review of contraception prescribing by GPs showed that there was continued use of the combined oral contraceptive pill rather than long-acting reversible contraception, which could suggest an ongoing need for EC as a result of poor adherence to shorteracting methods. ${ }^{11}$

In conclusion, it appears that when it comes to EC management there is a mismatch between the desired source of information, the actual source of information and the intermittent way this information is conveyed to women. It still remains unknown where and how women are obtaining information on EC, the credence invested in the information and its application. Neither is it known how GPs can better facilitate EC management and help women make informed decisions about their sexual and reproductive health. Further investigation is required so that women can achieve their desired fertility and health outcomes.

Competing interests: No relevant disclosures.

Received 30 Jul 2013, accepted 27 Nov 2013.

1 Marie Stopes International Australia. Real choices: women, contraception and unplanned pregnancy. Melbourne: Marie Stopes International Australia, 2008. http://www. mariestopes.org.au/images/stories/libraryfiles/ Real-Choices-Key-Findings.pdf (accessed Dec 2013).

2 National Collaborating Centre for Women's and Children's Health. Long-acting reversible contraception: the effective and appropriate use of long-acting reversible contraception. London: RCOG Press, 2005. http://www.nice.org.uk/ nicemedia/pdf/CG030fullguideline.pdf (accessed Dec 2013).

3 Finer LB, Henshaw SK. Disparities in rates of unintended pregnancy in the United States, 1994 and 2001. Perspect Sex Reprod Health 2006; 38 : 90-96.

4 Raymond EG, Trussell J, Polis CB. Population effect of increased access to emergency contraceptive pills: a systematic review. Obstet Gynecol 2007; 109: 181-188.

5 Hobbs MK, Taft AJ, Amir LH, et al. Pharmacy access to the emergency contraceptive pill: $a$ national survey of a random sample of Australian women. Contraception 2011; 83: 151-158.

6 Britt H, Miller GC, Henderson J, et al. General practice activity in Australia 2011-12. Sydney: Sydney University Press, 2012. http:// purl.library.usyd.edu.au/sup/9781743320181 (accessed Dec 2013).

7 Classification Committee of the World Organization of Family Doctors. ICPC-2: International Classification of Primary Care. 2nd ed. Oxford: Oxford University Press, 1998.

8 Britt H. A new coding tool for computerised clinical systems in primary care--ICPC plus. Aust Fam Physician 1997; 26 Suppl 2: S79-S82.

9 Britt H, Miller G, Bayram C. The quality of data on general practice - a discussion of BEACH reliability and validity. Aust Fam Physician 2007: 36: 36-40.

10 Hussainy SY, Stewart K, Chapman CB, et al. Provision of the emergency contraceptive pill without prescription: attitudes and practices of pharmacists in Australia. Contraception 2011; 83: 159-166.

11 Mazza D, Harrison C, Taft A, et al. Current contraception management in Australian general practice: an analysis of BEACH data. Med J Aust 2012; 197: 110-114. 